\title{
Blueprint for a Brain Health Clinic to Detect and Manage Early-Stage Cognitive Decline: A Consensus Exercise
}

Iracema Leroi ${ }^{1 *}$ (D), Charlotte Peel ${ }^{2}$, Rebecca Davenport ${ }^{3}$, Ross Dunne ${ }^{4}$, Louise Ebenezer ${ }^{5}$, Mahesh Gopakumar $^{6}$, Vachagan Krishnaswami ${ }^{7}$, Jane Lumsden ${ }^{8}$, Helen Martin ${ }^{9}$, Jane Price ${ }^{10}$ and Wilby Williamson $^{1,11}$

${ }^{1}$ Global Brain Health Institute, Trinity College Dublin, Ireland

${ }^{2}$ Neurology Academy, UK

${ }^{3}$ Manchester Royal Infirmary, UK

${ }^{4}$ Greater Manchester Dementia Research Centre Manchester, UK

${ }^{5}$ Princess of Wales Hospital, Cwm Taf Morgannwg University Health Board, Wales

${ }^{6}$ NHS Training Scheme, Greater Manchester Mental Health Foundation Trust, UK

${ }^{7}$ Department of Geriatrics, Antrim Hospital, Northern Health and Social Care Trust, UK

${ }^{8}$ National CJD Research and Surveillance Unit, University of Edinburgh, UK

${ }^{9}$ Ashville Surgery, UK

${ }^{10}$ Parkinson's Nurse Advanced Practitioner, Powys Teaching Health Board, Bronllys Hospital, UK

${ }^{11}$ Radcliffe Department of Medicine, Division of Cardiovascular Medicine, Oxford Cardiovascular Clinical Research

Facility, University of Oxford, UK

\begin{abstract}
Background: It is possible that about $30 \%$ of all dementia is preventable by addressing many of the modifiable health and lifestyle risks important for overall physical health. Currently, people in the pre-dementia or very early dementia stage who are referred to Memory Assessment Services (MAS) in the UK receive minimal, if any, support and/or intervention. They are typically referred back to primary care until the full syndrome of dementia emerges. This represents a lost opportunity to modify the trajectory of the condition, intervene with disease modifying therapies (DMTs) when available, and delay the onset of a full dementia syndrome.
\end{abstract}

Objective: We aimed to develop a blueprint for a pragmatic 'Brain Health Clinic' (BHC) that can be implemented alongside, or in conjunction with, conventional MAS.

Methods: Using modified consensus methods, an interdisciplinary task force of clinicians with experience in the diagnosis and care of people with cognitive impairment and dementia, met on several occasions to review existing evidence, share clinical experience, and propose a model for a pragmatic, 'real life' BHC, as an extension of, or embedded within, a current MAS.

Results: The BHC is a systems-based, integrated care approach that uses existing resources, and can be developed by reconfiguring the way current MAS are provided. It can support people with early-stage cognitive impairment to remain well for longer, potentially changing outcomes. The practical, evidencebased and user-friendly blueprint is available as a free online tool (depicted in figures throughout this article). It sets out a vision for managing early-stage cognitive decline using a 'preempt-prevent' approach that maximizes brain health and quality of life for the person at risk and their families. It sets the stage for implementation of validated, clinically useful biomarker batteries and DMT to be introduced when available, fostering personalized cognitive healthcare.

Conclusion: Adapting existing services to address neurodegenerative cognitive decline in the very earliest stages is a key intervention for secondary prevention of dementia.

\section{Keywords}

Brain health, Dementia prevention, Brain health clinic, Service model, Modifiable risks, Lifestyle

\footnotetext{
*Corresponding author: Iracema Leroi, Global Brain Health Institute, Trinity College Dublin, School of Medicine, Lloyd Building, Dublin 2, Republic of Ireland, Tel: +353-858600291, Email: Iracema.Leroi@tcd.ie

Accepted: March 03, 2020

Published online: March 05, 2020

Citation: Leroi I, Peel C, Davenport R, et al. (2020) Blueprint for a Brain Health Clinic to Detect and Manage Early-Stage Cognitive Decline: A Consensus Exercise. J Neurodegener Disord 3(1):54-64
} 
Citation: Leroi I, Peel C, Davenport R, et al. (2020) Blueprint for a Brain Health Clinic to Detect and Manage Early-Stage Cognitive Decline: A Consensus Exercise. J Neurodegener Disord 3(1):54-64

\section{Background}

According to population and epidemiologic analyses, the number of people with dementia globally is continuing to grow. There are over 46 million people living with dementia worldwide [1] and one in twenty people are diagnosed with 'young onset' dementia [2]. In the United Kingdom (UK), there are currently over 900,000 people living with dementia at a cost of $£ 26$ billion per annum [3]. Treatments are currently confined to therapies that ameliorate symptoms for a short period in a sub-group of people. There is hope that by 2025, disease modifying therapies (DMTs) for Alzheimer's disease $(A D)$, the commonest cause of dementia, will be available.

Theoretically, about $30 \%$ of all dementia in the population is preventable by reducing many recognized health risks, including hypertension, type 2 diabetes, obesity, smoking, and sedentarism [4], as well as less commonly known factors such as midlife hearing loss, depression and social isolation [4], alcohol misuse [5], head-injury and delirium [6]. Preventative measures through lifestyle and risk factor modification [4] should be aligned with new pharmacological advances that are leading to DMTs in [7], as well as new biomarker-based diagnostic criteria [8] for, AD. Collectively, this presents a compelling case to review our existing model for dementia diagnostic services and to adopt a fresh approach to the diagnosis and management of AD.

This blueprint for a pragmatic Brain Health Clinic (BHC), developed through a consensus process of clinical stakeholders, takes these drivers into consideration and presents a model for detection, diagnosis and management of cognitive impairment in the pre-dementia or very early stage of dementia, with a focus on secondary prevention and intervention. This blueprint complements and extends existing, and more detailed, guidance on the infrastructure required for administration of DMT, when licensed [7]. The blueprint has been created as a free interactive online tool (https:// dementiaacademy.co/2019/11/28/a-practical-model-for-dementia-prevention/) to support clinicians seeking to develop a $\mathrm{BHC}$ in their locality.

\section{The Need to Reconfigure Memory Assessment Services (MAS)}

Memory Assessment Services (MAS), initially established as university-based medication-management clinics in the UK, were introduced to the National Health Service (NHS) in the 1990's when cognitive enhancing drugs were first licensed for AD. Over 200 MAS now exist in the UK as an integral part of most later life mental health services, although very few focus on the prevention of progression from prodromal dementia to the full clinical syndrome of dementia.

At present, patients assessed in the early stages of cognitive decline (i.e. subjective memory complaints (SMC), mild cognitive impairment $(\mathrm{MCl})$, or pre-clinical and prodromal $A D)$, are generally referred back to primary care without further support or intervention. For some, their condition will stabilize or even improve; however, a significant proportion will progress to a full dementia syndrome. At this point, re-referral to MAS for a diagnosis and management plan is often initiated. Unfortunately, some people might not be referred back to a MAS for intervention even if progression has occurred. Furthermore, the diagnosis of $\mathrm{MCl}$ or very early stage dementia in MAS is often inconsistent and may not be based on updated biomarker-based diagnostic criteria [8]. This is despite recent evidence that the use of such testing changes treatment plans for up to $30 \%$ of MAS attendees [9].

For individuals at risk of dementia, a biomarker-based diagnosis of underlying disease may make a significant difference to life planning and the desire for follow-up. Vos, et al. [10] have demonstrated that those with both positive amyloid and neuronal injury biomarkers have a 20 times higher risk (60\% versus $5 \%$ ) of progression from prodromal disease to dementia over a three-year period. By intervening with 'brain-healthy lifestyle' practices during this three-year period there is an opportunity to potentially modify this outcome, as well as to support a proactive and timely approach to diagnosis and care at the first moment it may be required.

A recent clinical audit conducted in three UK-based $\mathrm{Na}$ tional Health Service (NHS) MAS in Manchester informed part of the development of this blueprint. The audit, a snapshot of 379 newly referred and follow-up patients over a threemonth period, revealed significant variability in the assessment, diagnosis and management of the 43 (11\%) people presenting with $\mathrm{MCl}$ or early-stage dementia, both within and across the three sites [11]. Data were gathered on the diagnosis of $\mathrm{MCl}$ and its sub-typing (if done), information needed to track 'at-risk' patients, the nature of any follow-up plans, and whether patients had transitioned to dementia. The findings revealed low adherence to evidence-based diagnostic standards, and inconsistent use of biomarkers (neuroimaging and fluid) and predictive risk models. There was inconsistent subtyping of $\mathrm{MCl}$ in $50 \%$, and only $26.7 \%$ of cases had documentation of accepted criteria. Furthermore, $32.6 \%$ of the follow-up patients had been identified with vascular-related $\mathrm{MCl}$, and there was no evidence that their vascular risk factors had been addressed [12].

Over the past few years in the UK, there has been a push to decrease MAS involvement in the post-diagnostic care of those at risk of, or with dementia, and there has been a corresponding increase in the outsourcing of services to non-medical providers. Since the prevalence of $\mathrm{MCl}$ is estimated at onethird of the population aged over 60-years [13], and the rate of conversion to dementia from $\mathrm{MCl}$ is estimated at about 6 to $15 \%$ per year [14], there is a strong case to consider a new approach for how early-stage cognitive decline should be managed. Recent health economic modelling has suggested that a pre-clinical diagnosis of AD and treatment with DMTs could cost health services several million per year; however, the future health and social care (particularly informal) cost savings due to DMTs could be in the billions [15]. This BHC blueprint, using a pragmatic systems-based approach that stretches across primary and secondary care (tertiary care, if indicated), is proposed as a potential solution by extending or adapting currently operating MAS. 


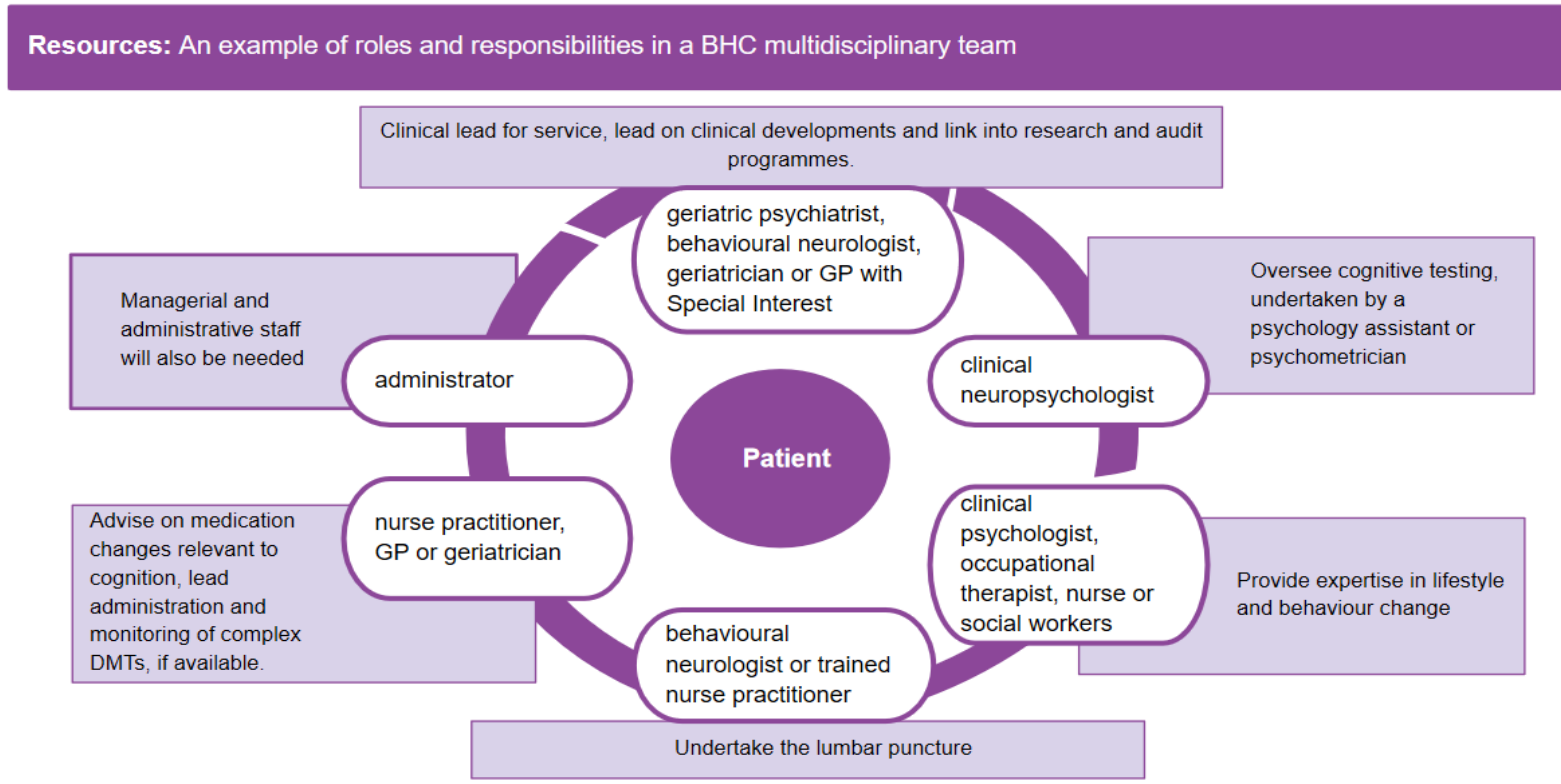

Figure 1: Roles and responsibilities in a Brain Health Clinic multidisciplinary team.

\section{Objectives}

The overall aim of a $\mathrm{BHC}$ is to lower the prevalence of dementia in the community that it serves. At present, many health services prioritize reactionary approaches and crisis management over preventative support. By risk stratifying all those with early-stage cognitive decline, individualized care is more achievable. This can equip those at low risk with the information and support they need to live "brain-healthy' lives and can provide those at high risk with early interventions, enabling them to live better for longer (Figure 1).

The specific objective of this blueprint is to outline an approach to early-stage cognitive decline from a secondary prevention perspective, and to maximize brain health and quality of life for those experiencing cognitive decline. The blueprint depicts an integrated service model which is adaptable to local service configuration, utilizes the latest validated assessment methods, and can be emulated nationally in most contexts.

\section{Method}

Following the MAS audit [11] to establish a baseline of activity, we used an iterative approach in four stages to develop our blueprint, as outlined in Table 1. We formed a task force involving the expertise of 32 delegates of the 2019 Dementia Masterclass (hosted by Neurology Academy, Sheffield, November 2018) in dialogue-based methods to elicit specialist knowledge and anecdotal clinical experience. From the outset, we established that the BHC blueprint would be based on practical, clinical experience and the emerging evidence base. Health economic aspects were beyond the remit of the project, and wider consultation with patients and care partner stakeholder groups have yet to be conducted during an implementation phase.

\section{Results}

The collective input of the taskforce resulted in a blueprint for the BHC, outlined below and depicted in screen shots of the online $\mathrm{BHC}$ tool.

\section{Purpose of the Brain Health Clinic (BHC)}

- To correctly diagnose patients with early-stage cognitive decline according to updated diagnostic classifications, including the application of biomarker information.

- To identify which patients are at risk of progressing to dementia, compared to those whose condition will remain static or even improve (i.e. risk stratification).

- To prevent and slow the rate of transition from cognitive decline to dementia using varied interventions (i.e. risk modification and disease modification).

- To provide resources to patients and their families about brain health, the risks of dementia and how personal interventions (i.e. lifestyle changes) might alleviate such risks.

\section{Approach to the Brain Health Clinic (BHC) and team}

The $\mathrm{BHC}$ is designed as a pragmatic, integrated care approach that can be embedded into existing services or work in collaboration or consultation with external services [16]. It can seamlessly incorporate the support and expertise of primary through to tertiary care. The BHC blueprint can be adapted to any one of the three integrated care approaches. Embedded approaches usually involve different members of a team working in the same physical space, where consultative or collaborative approaches may involve pro- 
Table 1: Outline of method for developing the blueprint for a Brain Health Clinic.

\begin{tabular}{|c|c|}
\hline Project timeline: & 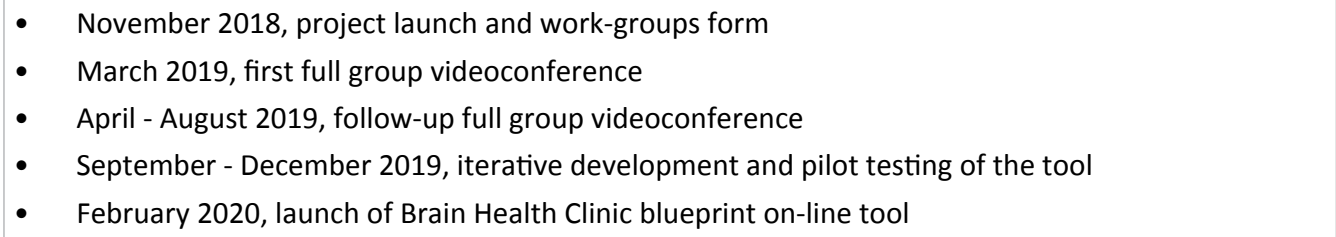 \\
\hline Setting and procedures: & $\begin{array}{l}\text { - } \begin{array}{l}\text { Baseline clinical audit of three representative Memory Assessment Services in Manchester, UK to } \\
\text { - }\end{array} \\
\text { Initial contact with professional stakeholders via a two-day Dementia Academy clinical masterclass } \\
\text { hosted by Neurology Academy (www.neurologyacademy.org), Sheffield, UK (11/2018) to form a } \\
\text { project task force } \\
\text { - Project orientation session introducing aims, method and timeline } \\
\text { - Five delegate workgroups formed; each assigned a sub-section of the tool } \\
\text { - } \quad \text { Forkgroup video-linked meeting every two months to draft the blueprint } \\
\text { - Sheffield, UK, June } 2019 \\
\text { - } \quad \text { Fixth and seventh iterations developed by the workgroup }\end{array}$ \\
\hline Task force participants & 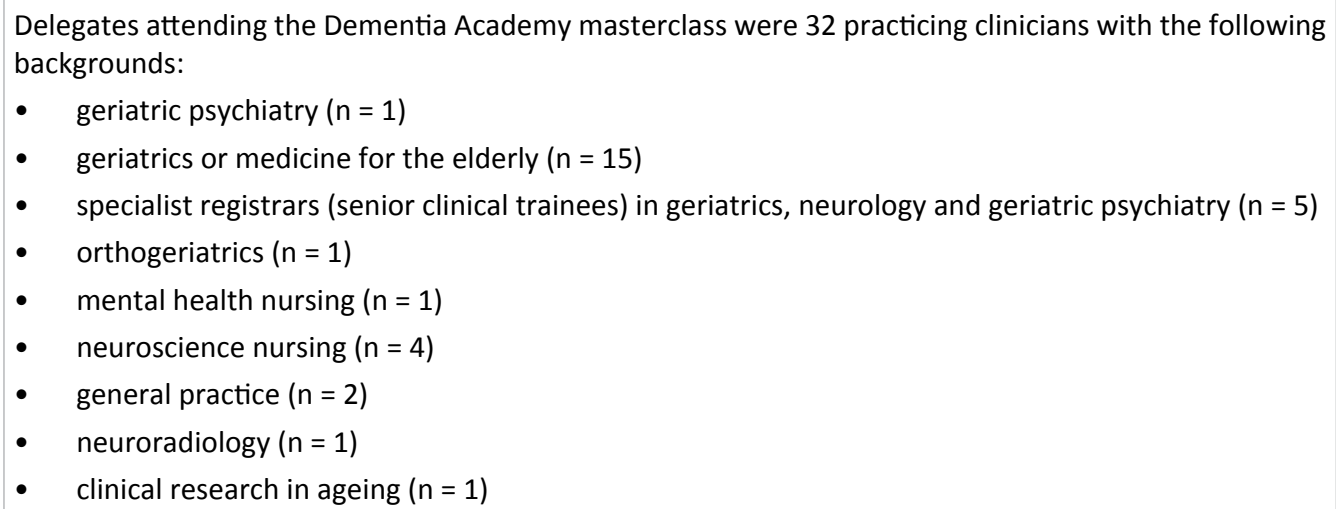 \\
\hline Facilitators: & $\begin{array}{l}\text { - The project lead and Dementia Academy director (IL) facilitated the group sessions and video-linked } \\
\text { meetings } \\
\text { - } \quad \text { Each workgroup was facilitated by a Dementia Academy faculty member } \\
\text { - } \quad \text { Each workgroup appointed a designated lead to coordinate work }\end{array}$ \\
\hline $\begin{array}{l}\text { Data collected to develop the } \\
\text { blueprint for the Brain Health } \\
\text { Clinic: }\end{array}$ & $\begin{array}{l}\text { - Stage 1: Audit of MCI diagnosis and patient journey in NHS UK MAS's (11) } \\
\text { - Stage 2: Expert consultation, divided into five work workgroups to develop the components of the } \\
\text { BHC: } \\
\text { - } \quad \text { Aim and ethos of the BHC } \\
\text { - } \quad \text { Steps in a diagnostic work-up to establish a meaningful diagnosis of prodromal dementia, including } \\
\text { - the sub-type, and risk stratification } \\
\text { - } \quad \text { sypes of interventions (pharmacological and non-pharmacological) and the infrastructure to } \\
\text { - } \quad \text { Stage 3: Consultation with experts of the draft tool } \\
\text { - Stage 4: On-line launch of the tool, Blueprint for a BHC }\end{array}$ \\
\hline
\end{tabular}

fessionals from different services all working together in a systems-based manner towards a common goal, stretching across tiers of the health and social care system. A key focus of the $\mathrm{BHC}$ is to support and empower individuals to navigate around existing health and social care systems, enabling them to tailor their healthcare to meet their needs and achieve the outcomes they choose.

\section{Brain Health Clinic (BHC) team}

The core BHC team comprises professionals from different disciplines across the primary to tertiary care spectrum, each with different roles and responsibilities (Figure 1). Whilst the specialist assessments and initial interventions will be undertaken by members of the core team, they will link in with a range of services and providers both internal and external to the host organization (usually a local MAS). The 
team can meet in person or virtually for multi-disciplinary team meetings (MDTs). Communication among professionals involved and movement of the patient between components can be regulated by a 'brain health guide', MDT coordinator or a non-specialist case manager.

\section{Patient inclusion criteria for the Brain Health Clinic (BHC)}

The BHC will accept referrals directly from primary care, secondary acute care (in- or out-patient), as well as conventional MAS.

- Adult age (above 18)

- Self- or informant-reported cognitive complaint

- Subjective or objective cognitive impairment not meeting criteria for a clinical syndrome of dementia (i.e. preserved or relatively preserved independence in functional abilities (defined by 'activities of daily living' ${ }^{b}$

- First-degree relative of people with dementia or diagnosis of a cognitive neurodegenerative disorder

- Episode of delirium within the past six months

alt is expected that almost all referrals will be people of the age of 55, but clinicians should be vigilant for young-onset dementia.

${ }^{b}$ It is expected that most referrals will have some degree of subtle functional impairment.

\section{Exclusion Criteria for the Service}

- Non-degenerative cognitive impairment due to another identified cause at the point of referral (e.g. depression, alcohol and drug misuse), which requires first line treatment first line; if the cognitive complaint persists after effective treatment of primary illness, the referral can be considered.

\section{Components of the Brain Health clinic (BHC)}

The blueprint illustrates the component parts of the BHC, giving clear recommendations on how to operationalize the service model, including information about existing resources. The BHC has four main components (outlined below), which are identification (Figure 2), assessment (Figure 3), patient journey (in three intervention streams) (Figures 4, Figure 5, Figure 6), and ongoing care (Figure 7).

Identification: This involves assessing and identifying appropriate referrals, using assessment tools (Figure 2). At the point of referral, it is expected that a basic medical and cognitive work-up will have been completed to rule out reversible causes for cognitive complaints (e.g. depression and thyroid dysfunction) and to establish that the patient is in the pre-dementia stage. The initial tier of cognitive testing will depend on whether the patient is referred directly from primary care (e.g. screening tools such as the General Practitioner Assessment of Cognition (GPCOG; [17]) and the 6-item Cognitive Impairment Test (6CIT; [18]) may be useful) or a secondary care MAS (e.g. Addenbrooke's Cognitive Evaluation-III,ACE-III; [19] or the Montreal Cognitive Assessment, MoCA; [20]). A staging tool should also be applied to ascertain whether the patient falls into the preclinical or prodromal stage of dementia (i.e. Clinical Dementia Rating Scale (CDR) [21] score of 0 to 0.5 ).

Assessment: This involves a detailed assessment including biomarkers and risk detection modelling to ensure sub-typing and prognosis of early stage cognitive decline (Figure 3). This includes clinical, lifestyle, behavioral, functional and cognitive assessments as well as biomarker detection. The outcome of the assessment will enable patients to be assigned to one of three risk-based 'streams' exemplified in the 'patient journey'

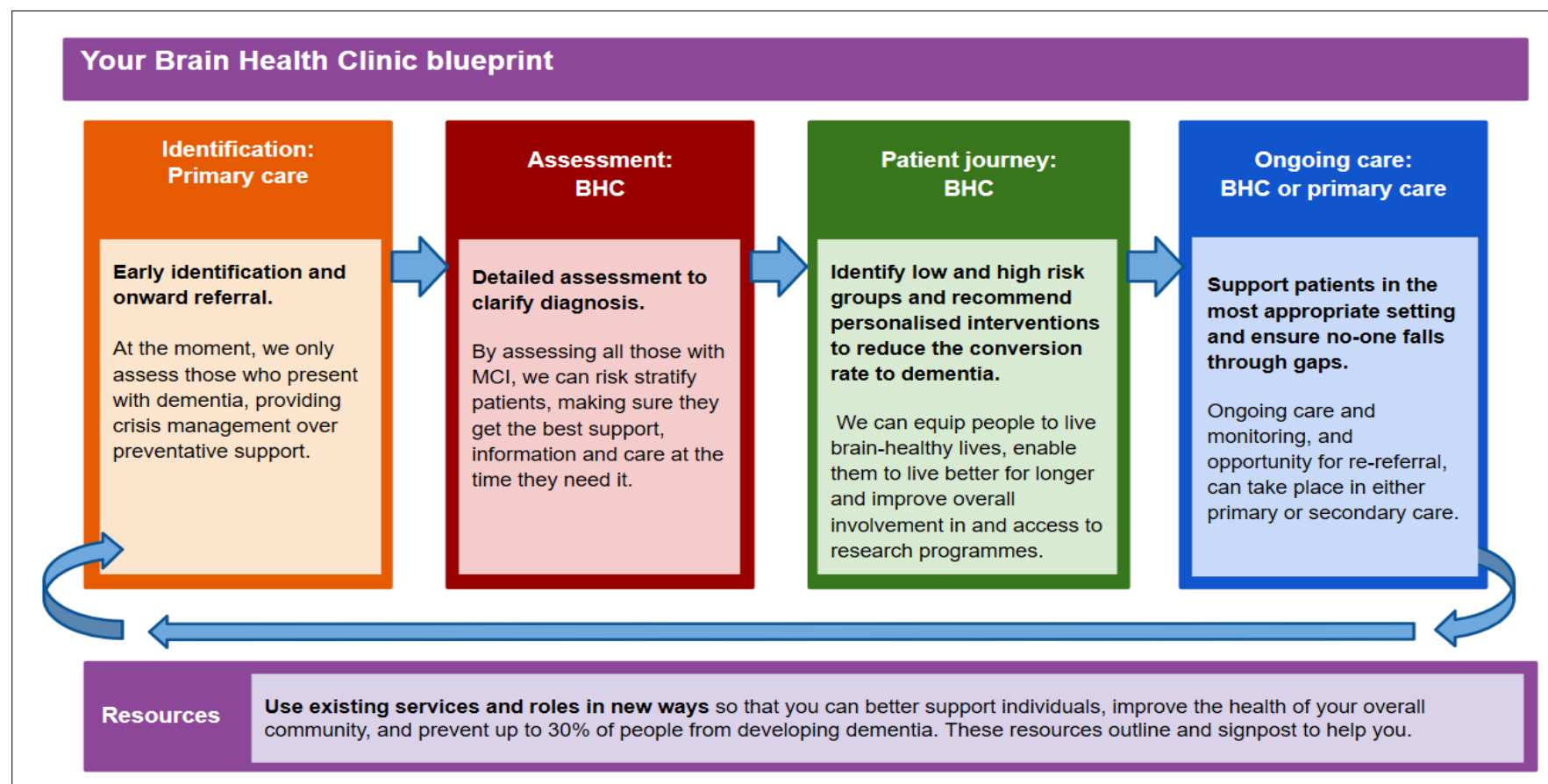

Figure 2: The Brain Health Clinic blueprint overview as depicted in the online tool. 


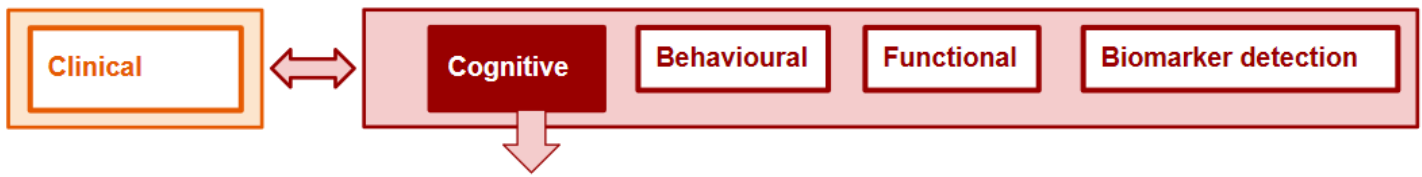

(Initial assessment: Global cognitive evaluation across different cognitive domains. Carried out at clinical assessment.)

Further assessment if $\mathrm{MCl}$ detected to striate into risk categories and ascertain if higher risk for progression to dementia due to Alzheimer disease: A feasible neuropsychological battery may be:

- $\quad$ The Repeatable Battery for the Assessment of Neuropsychological Status (RBANS) (4)

- The Free and Cued Selective Reminding Test (FCSRT) (5) especially beneficial in computerised form on a tablet/ laptop.

- The Cambridge Neuropsychological Test Automated Battery (CANTAB, Cambridge Cognition) (6) - a tablet-based cognitive test battery that captures the domains of attention, episodic memory, processing speed, working memory and executive function

Note: There is no agreed protocol; this recommendation is based on: protocol from Prevention of Alzheimer's Disease (EPAD) study 4, the Repeatable Battery for the Assessment of Neuropsychological Status (RBANS), the NIH EXAMINER/Toolbox 5 and additional tests of reaction time, processing speed, conceptual shifting, selective attention, allocentric spatial memory, paired-associate learning and navigation in egocentric space.

Identification

Assessment

Patient journey

Ongoing care

Resources

Figure 3: Example of one tab of the Brain Health Clinic tool's 'Assessment' section showing detail of the cognitive assessment in the BHC.

BHC patient journey (overview)

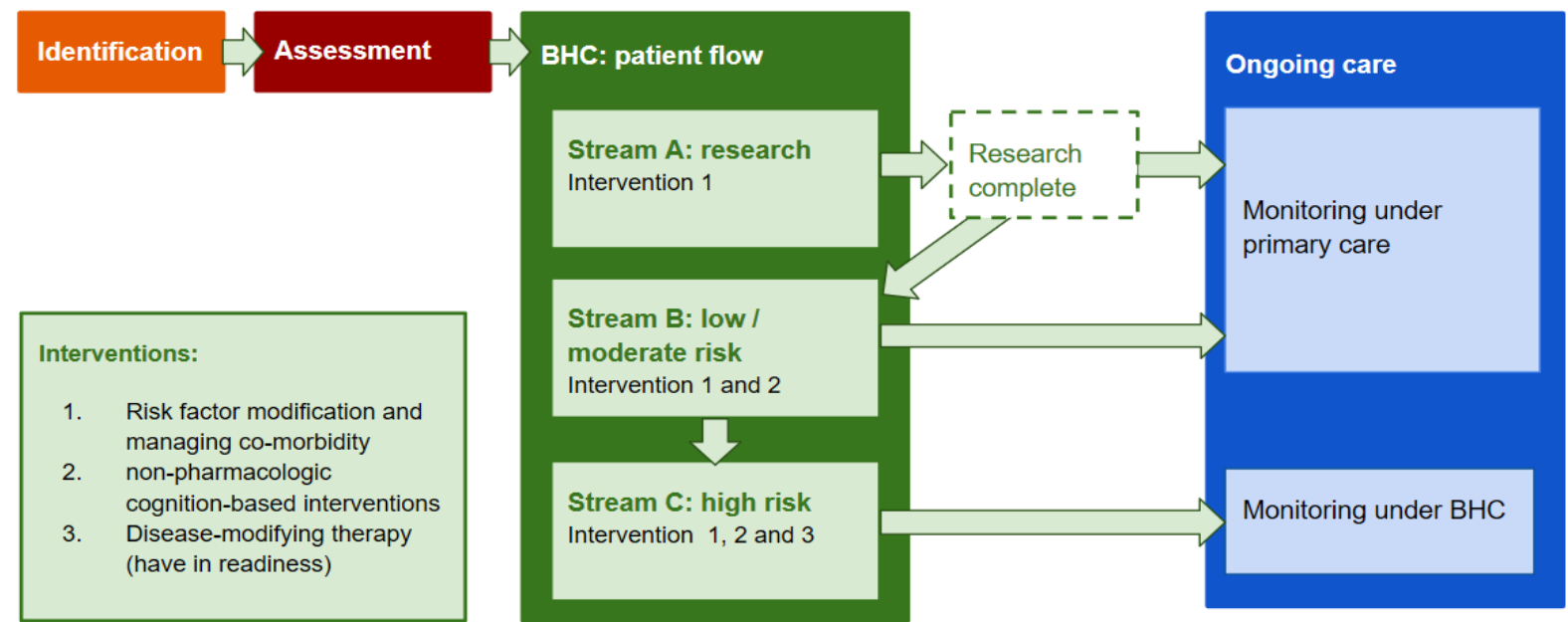

Identification

\section{Assessment}

Patient journey

Ongoing care

Resources

Figure 4: An overview of the patient journey from identification through to ongoing care, including the risk streams as taken from the blueprint tool. All panes navigate to the relevant sections within the online tool.

(Figures 4, Figures 5, Figures 6).

Clinical and lifestyle profile: Here, key potentially reversible risk factors are ascertained to inform risk-based decision making, and can include evaluations of vascular health status (e.g. diabetes, hypertension), sensory function (i.e. hearing and vision [22]), mental health status (e.g. depression), so- cial circumstances (e.g. isolation, loneliness, support network [23]), and lifestyle profile (e.g. activity, diet).

Cognitive profile: More detailed neuropsychological testing may include Repeatable Battery for Neuropsychological Status (RBANS; [24]), Free and Cued Selective Recall Test (FCS- 


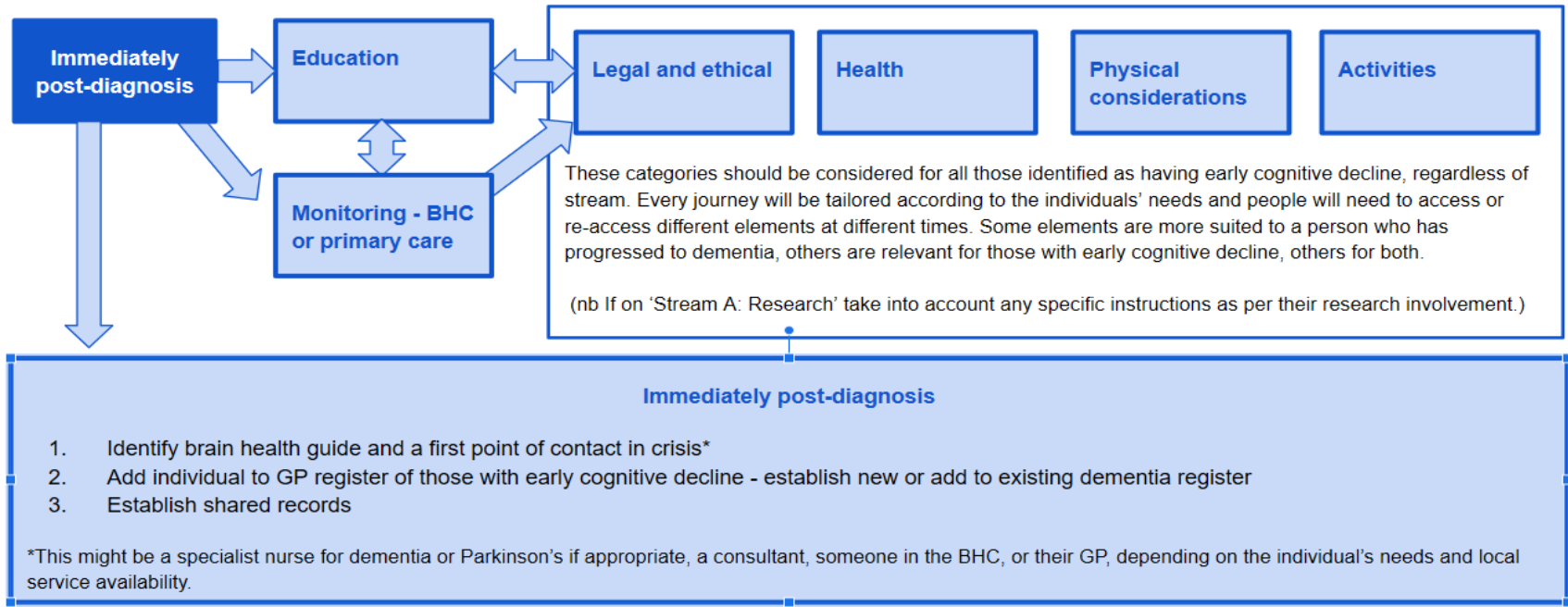

Identification Assessment Patient journey Ongoing care

Figure 7: Ongoing care is separated into various themes to assist navigation.

personal finances and using new personal technology) which may be indicators of impending dementia [33]. The Amsterdam IADL scale has undergone international validation studies $[34,35]$.

Biomarker detection: Details of an approach to brain health management in preparation for use of the new DMTs has already been outlined in detail by the Edinburgh Consensus initiative [7] and will thus not be repeated in full here. Instead, key biomarkers, as recommended under the International Working Group (IWG-2) criteria for AD [36] are briefly outlined. The use of biomarkers in the diagnosis of $A D$ in the pre-dementia stage has altered the characterization of $A D$ from being a syndrome-based diagnosis to a biologically based diagnosis.

Biomarkers include those involving $\beta$ amyloid $(A)$ deposition, pathologic tau $(T)$, and neurodegeneration $(N)$, or the ATN classification, detected by fluid and neuroimaging measures. However, the 'biological' diagnosis of $A D$ is recommended for research settings and is not yet widely used clinically, although the UK's NICE guidance (2018) recommends lumbar puncture for the diagnosis of $A D$ in people with $\mathrm{MCl}$ whose diagnosis may be unclear [37]. Finally, genetic testing for $A D$ risk using the apolipoprotein (APOE) genotype is not widely recommended in clinical settings despite being more readily available. Practice guidelines for the use of APOE testing have been published [38].

$\boldsymbol{\beta}$ amyloid $(\mathrm{A})$ and pathological tau $(\mathrm{T})$ deposition: These can be detected either by CSF lumbar puncture [39] or PET scans with amyloid or tau tracers $[40,41]$. Additionally, detection in elevated CSF levels of neurofilament light chain (NFL) protein, a marker for neuroaxonal damage due to $A D$ may add to the sensitivity and specificity of the biological diagnosis [42]. Its levels are elevated in the CSF of patients with $A D$ and other neurological conditions as compared with healthy controls. A recent meta-analysis by Olsson, et al. recommends using CSF Aß42, T-tau, P-tau and NFL levels as a panel of diagnostic biomarkers for $A D$ in both clinical practice and research [42].

Neurodegeneration $(\mathbf{N})$ : This can be detected by structural neuroimaging using magnetic resonance imaging (MRI) to detect such changes as medial temporal atrophy, suggestive of $A D[43,44]$ and changes indicative of cerebrovascular disease (quantified using Fazekas scores).

Assigning the patient intervention stream ('patient journey'): (Figures 4, Figures 5 and Figures 6) This involves stratifying into three 'streams' according to the patient's risk of progressing to dementia (low vs high) and being offered three multimodal intervention approaches designed to slow or even prevent the transition from early stage cognitive decline to dementia. Patients can move among streams if their risk profile changes due to successful risk modification. Additionally, the interventions are tailored to the individual's requirements and preferences.

The three streams are: A. Research stream, for those meeting eligibility criteria for ongoing research studies and who are interested and willing to participate. In the UK, potential participants can sign up to a research register (www.joindementiaresearch.nihr.ac.uk); B. Low/moderate risk stream to receive interventions 1 and 2 ; and $C$. High risk stream to receive interventions 1, 2 and 3 . The nature of the interventions is outlined below:

Intervention 1: Lifestyle risk factor modification, medication rationalization, and managing co-morbidity (includes both pharmacologic and non-pharmacologic interventions pertaining to risk factors) will be offered to patients in the high risk and low risk streams. 
Lifestyle risk factor modification: Support to make relevant lifestyle changes to foster brain health and modify risk will be offered, with focus on nutrition, alcohol, sleep, smoking and movement. Emerging evidence from international studies such as the FINGER trial [45] suggests that multi-modal interventions simultaneously targeting vascular and other lifestyle changes in older people with higher risk of AD (i.e. APOE4 positive) may have a slower rate of cognitive decline. Ideally, the lifestyle changes should be framed by evidence-based behavioral psychology approaches to ensure uptake and long-term adherence with the changes.

Medication rationalization: A key aspect at this stage will be a thorough review of medications to ensure that they are not the cause of the cognitive impairment, nor are increasing the risk of cognitive decline. Benzodiazepines, opioid analgesics, certain psychotropics, and medications with high anticholinergic loads should be reviewed and rationalized, if possible $[46,47]$.

Managing co-morbidity: Addressing medical comorbidity, particularly vascular risks, sensory functioning (hearing and vision impairment) and other potential physical causes of cognitive decline need to be carefully addressed, establishing a baseline for ongoing monitoring of medical risk factors and potential modification.

Intervention 2: Non-pharmacologic cognition-based interventions, such as brain training and cognitive stimulation, have been evaluated in the context of dementia due to $A D$ and $\mathrm{MCl}$ and have demonstrated improvements in memory in healthy older people and people with $\mathrm{MCl}$ [48-51]. The theoretical basis for such cognition-based interventions includes the restoration or activation of compensatory brain mechanisms to improve specific cognitive functions or adapting training to accommodate cognitive impairment by using contextualized perspectives [52].

Intervention 3: DMTs, which have yet to be clinically licensed for therapeutic use, will be the final intervention mode for the BHC as they need to be administered in the very earliest phases of the disease before neuronal degeneration is widespread. These therapies will likely involve different mechanisms of actions such as anti-amyloid and anti-tau antibody-based agents, and anti-inflammatory agents inhibiting pathological cascades.

Ongoing care: Here the blueprint outlines suggested monitoring and follow-on care, either in primary care or under the $B H C$, depending on the patient's risk of developing dementia (Figure 7). Those in the low risk stream and who have less chance of conversion to dementia within three to five years may have less frequent follow-up (i.e. every two years). In contrast, those in the higher risk stream will have more frequent follow-up, at least every six months, with the potential for flexibility should progression appear to be faster or slower. Initially this will be clinic-based, but, as remote monitoring of continuous functions with clinical significance emerge (i.e. using passive and semi-passive monitoring systems of daily functions and activities), such monitoring will be home-based or even self-regulated.

\section{Discussion}

We have presented a proposal for a pragmatic, integrated care BHC that can be developed as an extension of, or adaptation to MAS, using existing resources and services. This represents a step forward in the clinical approach to dementia which needs to move beyond diagnosing and supporting people with existing dementia. It urgently needs to focus on prevention, delaying the onset of dementia, and very early identification of individuals at risk of progressing from early-stage cognitive decline to dementia. This can best be done in the setting of a BHC using an integrated or multidisciplinary care approach.

A key challenge in developing a $\mathrm{BHC}$ is the need to strike a balance between the emerging evidence regarding risk stratification and risk modification, and what is practical, cost-effective and feasible to undertake in standard clinical settings. Currently, no specific risk stratification tool has been approved for clinical use [53], although an increasing number of tools are being published and validated. Most tools include age, education, and measures of cognition and health, but It is increasingly likely that the most useful tools, once fully validated, will involve multivariable risk prediction models based on demographic, genetic, cognitive, health and lifestyle measures. Furthermore, while lifestyle and health status are emerging as powerful risk factors, apart from a few exceptions such as the FINGER trial [54] and ongoing efforts such as the European Prevention of Alzheimer Disease (EPAD) initiative [55], Level I evidence for risk modification is still lacking. Nonetheless, considering the long 'tail' of disease progression in the preclinical stage of $A D$ and other neurodegenerative conditions leading to dementia, it is prudent to adopt a common-sense approach by instituting changes in existing MAS.

As pointed out by the 2017 Lancet Commission [4], 'acting now on dementia prevention, intervention, and care will vastly improve living and dying for individuals with dementia and their families, and in doing so, will transform the future for society'. The next step will be to consult patient and family care stakeholders, and evaluate implementation, including economic modelling. Approaches such as this, which enable tailoring to the needs of the individual, are essential to ensure the best possible care for an aging population with enduring healthcare concerns, and can begin to address the prevalence of dementia in our society.

\section{Acknowledgement}

Sarah Gillett, Jamie McGregor, Jackey Spiers from the Neurology Academy, and participants of the UK's Dementia Academy Masterclass, (November 2018), Sheffield, UK. Lucy Colwill contributed to the task force.

\section{References}

1. (2015) World Alzheimer Report 2015: The global impact of dementia. An analysis of prevalence, incidence, cost and trends (ADI: London). Alzheimer's Disease International.

2. https://www.mayoclinic.org/diseases-conditions/alzheimers-disease/in-depth/alzheimers/art-20048356 
Citation: Leroi I, Peel C, Davenport R, et al. (2020) Blueprint for a Brain Health Clinic to Detect and Manage Early-Stage Cognitive Decline: A Consensus Exercise. J Neurodegener Disord 3(1):54-64

3. https://www.alzheimers.org.uk/about-us/policy-and-influencing/what-we-think/dementia-tax

4. Livingstone G, Sommerlad A, Orgeta V, et al. (2017) Dementia prevention, intervention and care. Lancet 390: 2673-2734.

5. Sabia S, Fayosse A, Dumurgier J, et al. (2018) Alcohol consumption and risk of dementia: 23 year follow-up of Whitehall II cohort study. BMJ 362: 2927.

6. Brayne C, Davis D (2012) Making Alzheimer's and dementia research fit for populations. Lancet 380: 1441-1443.

7. Ritchie CW, Russ TC, Banerjee S, et al. (2018) The Edinburgh Consensus: Preparing for the advent of disease-modifying therapies for Alzheimer's disease. Alzheimer's Research \& Therapy 9: 85.

8. McKhann GM, Knopman DS, Chertkow H, et al. (2011) The diagnosis of dementia due to Alzheimer's disease: recommendations from the National Institute on Aging-Alzheimer's Association workgroups on diagnostic guidelines for Alzheimer's disease. Alzheimers Dement 7: 263-269.

9. Rabinovici G, Gatsonis C, Apgar C, et al. (2019) Association of amyloid positron emission tomography with subsequent change in clinical management among medicare beneficiaries with mild cognitive impairment or dementia,' within 'Imaging Dementia Evidence for Amyloid Scanning (IDEAS). JAMA 321: 1286-1294.

10. Vos S, Xiong C, Visser PJ, et al. (2013) Preclinical Alzheimer's disease and its outcome: A longitudinal cohort study. Lancet Neurol 12: 957-965.

11. Leroi I, Smith C (2018) Project 5073-Service evaluation of the patient population with a diagnosis of mild cognitive impairment within Memory Assessment Services in Manchester. Greater Manchester Mental Health Foundation Trust.

12. Sabia S, Fayosse A, Dumurgier J, et al. (2019) Association of ideal cardiovascular health at age 50 with incidence of dementia: 25year follow-up of Whitehall II cohort study. BMJ 366: 14414.

13. Sachdev PS, Lipnicki DM, Kochan NA, et al. (2015) The Prevalence of mild cognitive impairment in diverse geographical and ethnocultural regions: The COSMIC Collaboration. PLOS ONE 10 e0142388.

14. Petersen RC, Roberts RO, Knopman DS, et al. (2009) Mild cognitive impairment: Ten years later. Arch Neurol 66: 1447-1455.

15. Anderson R, Knapp M, Wittenberg R, et al. (2018) Economic modelling of disease-modifying therapies in Alzheimer's disease. PSSRU.

16. Pontone GM, Dissanayaka N, Dobkin RD, et al. (2019) Integration and extension of specialty mental healthcare services to community practice in Parkinson disease. Am J Geriatric Psychiatry 27: 712-719.

17. Brodaty H, Kemp NM, Low LF (2004) Characteristics of the GPCOG, a screening tool for cognitive impairment. International Journal of Geriatric Psychiatry 19: 870-874.

18. Brooke P, Bullock R (1999) Validation of a 6-item cognitive impairment test with a view to primary care usage. Int J Geriatr Psychiatry 14: 936-940.

19. Hsieh S, Schubert S, Hoon C, et al. (2013) Validation of the Addenbrooke's cognitive examination III in frontotemporal dementia and Alzheimer's disease. Dement Geriatr Cogn Disord 36: 242-250.

20. Nasreddine ZS, Phillips NA, Bedirian V, et al. (2005) The Montreal Cognitive Assessment, MoCA: A brief screening tool for mild cog- nitive impairment. J Am Geriatr Soc 53: 695-699.

21. O'Bryant SE, Waring SC, Cullum CM, et al. (2008) Staging dementia using clinical dementia rating scale sum of boxes scores: A Texas Alzheimer's research consortium study. Arch Neurol 65: 1091-1095.

22. Maharani A, Dawes P, Nazroo J, et al. (2018) Visual and hearing impairments are associated with cognitive decline in older people. Age and ageing 47: 575-581.

23. Maharani A, Pendleton N, Leroi I (2019) Hearing impairment, loneliness, social isolation and cognitive function: Longitudinal analysis using English Longitudinal Study on Ageing. Am J Geriatr Psychiatry 66: 1130-1136.

24. Randolph C, Tierney MC, Mohr E, et al. (1998) The repeatable battery for the assessment of neuropsychological status (RBANS): Preliminary clinical validity. J Clin Exp Neuropsychol 20: 310-319.

25. Grober E, Sanders AE, Hall C, et al. (2010) Free and cued selective reminding identifies very mild dementia in primary care. Alzheimer Dis Assoc Disord 24: 284-290.

26. Kramer JH, Mungas D, Possin KL, et al. (2014) NIH Examiner: Conceptualisation and development of an executive function battery. J Int Neuropsychol Soc 20: 11-19.

27. Geda YE, Roberts RO, Knopman DS, et al. (2008) Prevalence of neuropsychiatric symptoms in mild cognitive impairment and normal cognitive aging: Population-based study. Arc Gen Psychiatry. 65: 1193-1198.

28. Ismail Z, Agüera-Ortiz L, Brodaty, et al. (2017) The Mild Behavioral Impairment Checklist (MBI-C): A rating scale for neuropsychiatric symptoms in Pre-Dementia Populations. J Alzheimers Dis 56: 929-938.

29. Sikkes SA, de Lange-de Klerk ES, Pijnenburg YA, et al. (2012) A new informant-based questionnaire for instrumental activities of daily living in dementia. Alzheimers Dement 8: 536-543.

30. Cummings JL (1997) The Neuropsychiatric Inventory: assessing psychopathology in dementia patients. Neurology 48: S10-S16.

31. Kaufer DI, Cummings JL, Ketchel P, et al. (2000) Validation of the NPI-Q, a brief clinical form of the Neuropsychiatric Inventory. J Neuropsychiatry Clin Neurosci 12: 233-239.

32. de Medeiros K, Robert P, Gauthier S, et al. (2010) The Neuropsychiatric Inventory-Clinician rating scale (NPI-C): reliability and validity of a revised assessment of neuropsychiatric symptoms in dementia. Int Psychogeriatr 22: 984-994.

33. Peres K, Helmer C, Amieva H, et al. (2008) Natural history of decline in instrumental activities of daily living performance over the 10 years preceding the clinical diagnosis of dementia: a prospective population-based study. J Am Geriatr Soc 56: 37-44.

34. Dubbelman MA, Verrijp M, Facal D, et al. (2020) The influence of diversity on the measurement of functional impairment: An international validation of the Amsterdam IADL Questionnaire in 8 countries. Neurons and Cognition.

35. Stringer G, Leroi I, Sikkes SAM, et al. (2019) Enhancing 'meaningfulness' of functional assessments: cultural adaptation of the Amsterdam IADL questionnaire for older UK people. International Psychogeriatrics.

36. Bruno Dubois, Howard H, Feldman, et al. (2014) Advancing research diagnostic criteria for Alzheimer's disease: The IWG-2 criteria. Lancet Neurol 13: 614-629. 
Citation: Leroi I, Peel C, Davenport R, et al. (2020) Blueprint for a Brain Health Clinic to Detect and Manage Early-Stage Cognitive Decline: A Consensus Exercise. J Neurodegener Disord 3(1):54-64

37. NICE guideline (2018) Dementia: Assessment, management and Dementia: assessment, management and support for people living with dementia support for people living with dementia and their carers and their carers.

38. Goldman J, Hahn S, Catania J, et al. (2011) Genetic counseling and testing for Alzheimer disease: Joint practice guidelines of the American College of Medical Genetics and the National Society of Genetic Counselors. Genet Med 13: 597-605.

39. Engelborghs S, Niemantsverdriet E, Struyfs H, et al. (2017) Consensus guidelines for lumbar puncture in patients with neurological diseases. Alzheimers Dement (Amst) 8: 111-126.

40. Berti V, Pupi A, Mosconi L (2011) PET/CT in diagnosis of dementia. Ann N Y Acad Sci 1228: 81-92.

41. Villemagne VL, Doré $V$, Bourgeat $P$, et al. (2017) $A \beta$-amyloid and Tau Imaging in Dementia Semin Nucl Med 47: 75-88.

42. Olsson B, Lautner R, Andreasson U, et al. (2016) CSF and blood biomarkers for the diagnosis of Alzheimer disease: A systematic review and metaanalysis. Lancet Neurol 15: 673-684.

43. Philip Scheltens, Laura van de Pol (2012) Atrophy of medial temporal lobes on MRI in "probable" Alzheimer's disease and normal ageing: Diagnostic value and neuropsychological correlates. J Neurol Neurosurg Psychiatry 83: 1038-1040.

44. PJ Visser, FRJ Verhey, PAM Hofman, et al. (2002) Medial temporal lobe atrophy predicts Alzheimer's disease in patients with minor cognitive impairment. J Neurol Neurosurg Psychiatry 72: 491-497.

45. Ngandu T, Lehtisalo J, Solomon A, et al. (2015) A 2-year multidomain intervention of diet, exercise, cognitive training, and vascular risk monitoring versus control to prevent cognitive decline in at-risk elderly people (FINGER): A randomised controlled trial. The Lancet 385: 2255-2263.

46. Green A, Oh E, Hilson L, et al. (2016) Anticholinergic burden in older adults with mild cognitive impairment. J Am Geriatr Soc 64: e313-e314.

47. Fox C, Livingston G, Maidment ID, et al. (2011) The impact of anticholinergic burdenin Alzheimer's dementia-the laser-AD study. Age and Ageing 40: 730-735.

48. Rojas GJ, Villar V, Iturry M, et al. (2013) Efficacy of a cognitive intervention program in patients with mild cognitive impairment. Int Psychogeriatr 25: 825-831.

49. Bahar-Fuchs A, Martyr A, Goh AM, et al. (2019) Cognitive training for people with mild to moderate dementia. Cochrane Database Syst Rev.

50. Lampit A, Hallock H, Valenzuela M (2014) Computerized cognitive training in cognitively healthy older adults: A systematic review and meta-analysis of effect modifiers. PLoS Med 11: e1001756.

51. Martin M, Clare L, Altgassen AM, et al. (2011) Cognition-based interventions for healthy older people and people with mild cognitive impairment. Cochrane Database Syst Rev.

52. Ylvisaker M, Hanks R, Johnson-Greene D (2002) Perspectives on rehabilitation of individuals with cognitive impairment after brain injury: Rationale for reconsideration of theoretical paradigms. J Head Trauma Rehabil 17: 191-209.

53. Tang EYH, Harrison SL, Errington L, et al. (2015) Current developments in dementia risk prediction modelling: An updated systematic review. PLoS One 10: e013618.

54. Kivipelto M, Solomon A, Ahtiluoto S, et al. (2013) The finnish geriatric intervention study to prevent cognitive impairment and disability (FINGER): Study design and progress. Alzheimers Dement 9: 657-665.

55. Solomon A, Kivipelto M, Molinuevo JL, et al. (2018) European Prevention of Alzheimer's Dementia Longitudinal Cohort Study (EPAD LCS): Study protocol. BMJ Open 8: e021017.

DOI: $10.36959 / 459 / 600$

Copyright: (C) 2020 Leroi I, et al. This is an open-access article distributed under the terms of the Creative Commons Attribution License, which permits unrestricted use, distribution, and reproduction in any medium, provided the original author and source are credited. 